\title{
L'orientation client du vendeur du point de vue du consommateur : les apports de la théorie de l'attachement
}

\author{
par Ghislaine Pellat, Fanny Poujol \\ et Béatrice Siadou-Martin
}

\section{Résumé}

La littérature en force de vente, essentiellement basée sur des études qualitatives menées au cours des années 80 , présente le concept d'orientation relationnelle comme la capacité du vendeur à contribuer à la satisfaction et à la pérennisation des relations commerciales. Notre étude vise à revisiter la nature de l'orientation client ou relationnelle des vendeurs sur la base de 67 récits de consommateurs recueillis à l'aide de la méthode des incidents critiques. Nous trouvons que l'orientation client est composée de deux facettes : la transmission d'expertise et la création de lien. Ces comportements relationnels du vendeur semblent jouer un rôle fondamental dans le processus de satisfaction client et dans la création de lien. Nous proposons une lecture originale de la construction de ce lien vendeur-client grâce à la théorie de l'attachement.

\section{Abstract}

The concept of customer orientation is presented in the sales force literature as the ability of the salesperson to contribute to the durability of the relationship. However, most of the studies dealing with customer orientation are based on qualitative studies conducted during the eighties. Our goal is to re-examine the nature of customer orientation of the salesperson and its consequences. On the basis of 67 customers' stories using the critical incident method, we found two facets : transmission of expertise and the creation of linkages. Salespeople's relational behaviours seem very important in the customer attachment process and in the creation of linkages. We offer an original view of the relationship development between the customer and the salespeople with the theory of attachment.

Le métier de vendeur a évolué ces dix dernières années, Bergadaà et Bernard (1998) qualifient ces mutations de véritable « révolution vente ». Les technologies de l'information et de la communication mises à disposition des commerciaux ont permis d'importants gains de productivité et une plus grande expertise de la clientèle. Loin d'éradiquer les vendeurs, ces outils ont enrichi sensiblement leur métier (Tanner et al, 2008). L'importance de l'orientation client du vendeur a été mise en lumière par les travaux sur l'orientation marché (Jaworski et Kohli, 1993). 
Pour ces chercheurs, l'orientation client de l'entreprise dont l'orientation client du vendeur est une composante, a un effet positif sur la fidélisation et au final sur les profits de l'entreprise. Le rôle relationnel du commercial s'est intensifié avec l'évolution du marketing vers le marketing relationnel où la vente ne se limite plus à un échange mais participe aussi à l'entretien d'une relation entre le vendeur et le client. La littérature sur les services (Crosby et al, 1990), en BtoC (Goff et al, 1997) et en BtoB (Biong et Selnes, 1996; Humphreys et Williams, 1996) accrédite le rôle du vendeur dans le développement de bonnes relations avec la clientèle. Le vendeur est considéré comme un acteur stratégique de la qualité de la relation, au cœur même de la confiance que le client peut accorder à l'entreprise (Garbarino et Johnson, 1999). Selon Moncrief et Marshall (2005), alors que l'entreprise cherche à construire et à entretenir des relations pérennes avec la clientèle, la performance relationnelle des commerciaux est aujourd'hui au premier plan. Cette performance est constituée par les comportements essentiels à la vente, à la création et à la préservation de la qualité de la relation dans le temps. Parmi les comportements clés des commerciaux mis en évidence par l'état de l'art, l'orientation client apparaît comme un déterminant majeur de la qualité et de la continuité de la relation client (Poujol, 2008).

L'orientation client du vendeur se caractérise par un comportement d'assistance du client dans sa décision d'achat (Saxe et Weitz, 1982). Un commercial est « orienté client » lorsqu'il s'engage dans des comportements conduisant à la satisfaction de son client à long terme plutôt que son propre intérêt à court terme (Kelley, 1992). L'orientation client recouvre ainsi un ensemble de comportements qui contribuent à la satisfaction à l'égard du vendeur et aussi plus largement, à l'égard de l'entreprise (Goff et al, 1997). Cependant, les travaux sur l'orientation client du vendeur sont essentiellement basés sur des données qualitatives collectées auprès de managers et de commerciaux, ces travaux datent des années 80 . Bergadaà $(2008)^{48}$ souligne l'intérêt de mieux cerner ce que recouvrent aujourd'hui les comportements relationnels du vendeur. Quels sont les éléments saillants d'un "bon " vendeur aux yeux des consommateurs ? Quels sont les comportements du vendeur qui conduisent à la satisfaction du client? Peuton identifier les étapes qui conduisent à une satisfaction de la relation par le client?

Aussi, à travers ce travail nous avons cherché à identifier les comportements saillants du vendeur qui participent à la construction de la relation plus ou moins éphémère. A partir d'une étude qualitative menée auprès de 67 consommateurs, nous revisitons le concept d'orientation client. En effet, cette notion ne semble pas recouvrir tous les comportements relationnels importants pour la clientèle. La méthode des incidents critiques est utilisée pour inciter les répondants à

48. Discussions lors du 24ème Congrès International de l'Association Française de Marketing et lors des VII Assises de la Vente intitulées « Sous le regard du client, les clefs pour comprendre les mutations de la fonction commerciale », 15 et 16 mai, Paris. 
se souvenir d'une situation particulièrement marquante avec un vendeur. Les résultats montrent que l'orientation relationnelle comporte divers aspects qui sont illustrés avec des verbatim.

Dans un premier temps, nous présentons une revue de la littérature sur le rôle du vendeur dans la relation client et exposons les principaux comportements relationnels relevés dans la littérature. Dans un deuxième temps, nous expliquons la méthodologie de la recherche choisie, justifiant le choix de la méthode des incidents critiques qui se révèle bien adaptée à notre question de recherche. Enfin, les principaux résultats de cette étude sont ensuite détaillés et rapprochés des apports académiques, et notamment de la théorie de l'attachement qui a en commun avec notre préoccupation, la création du lien, élément premier des relations. Nous terminons par des implications managériales et des voies futures de recherches.

\section{Revue de la littérature}

Notre revue de la littérature s'est tout d'abord centrée sur le rôle du vendeur dans la relation client et notamment dans la rencontre de service avant d'approfondir la notion d'orientation client du vendeur, ses antécédents, les conséquences, les concepts proches et ses limites.

\subsection{Le rôle du vendeur dans la relation client}

Au-delà des évènements ponctuels, les transactions constituent une suite d'échanges qui exercent des influences entre eux : ils peuvent se renforcer, se compenser ou s'inhiber. Ainsi la fidélité "évolue d'un point de vue centré sur la satisfaction des besoins vers un point de vue centré sur la relation laquelle s'exprime par l'engagement et la confiance que se témoignent les protagonistes de l'échange » (Moulins, 1998, p.67). L'examen de la littérature sur la relation laisse émerger trois caractéristiques principales : la réciprocité, l'engagement à tenir ses promesses ainsi que la confiance et ses conséquences (Grönroos, 1990 ; Morgan et Hunt, 1994).

Pour Dwyer et al, (1987), l'échange relationnel se déroule dans le temps, chaque transaction doit être envisagée dans la perspective de son historique et de l'anticipation de son futur. C'est ainsi l'interdépendance de ces transactions qui crée la dynamique de la relation dans le temps. Ces chercheurs utilisent la métaphore du mariage pour qualifier le développement de la dyade acheteurvendeur. Le rôle du personnel dans la relation entre l'entreprise et le client a été mis en évidence dans le domaine des services dès les années 80 . Les comportements du personnel font partie intégrante de la «servuction» et participent largement au succès de l'interaction entreprise-client. La rencontre ou plutôt les rencontres de service successives seraient cruciales en termes de 
qualité du service et de pérennisation de la relation client (Bitner et al, 1990) comme le montre la figure 1 suivante.

Figure 1 : Le rôle du vendeur dans la relation client adapté du modèle de Servuction,

(Eiglier et Langeard, 1987)

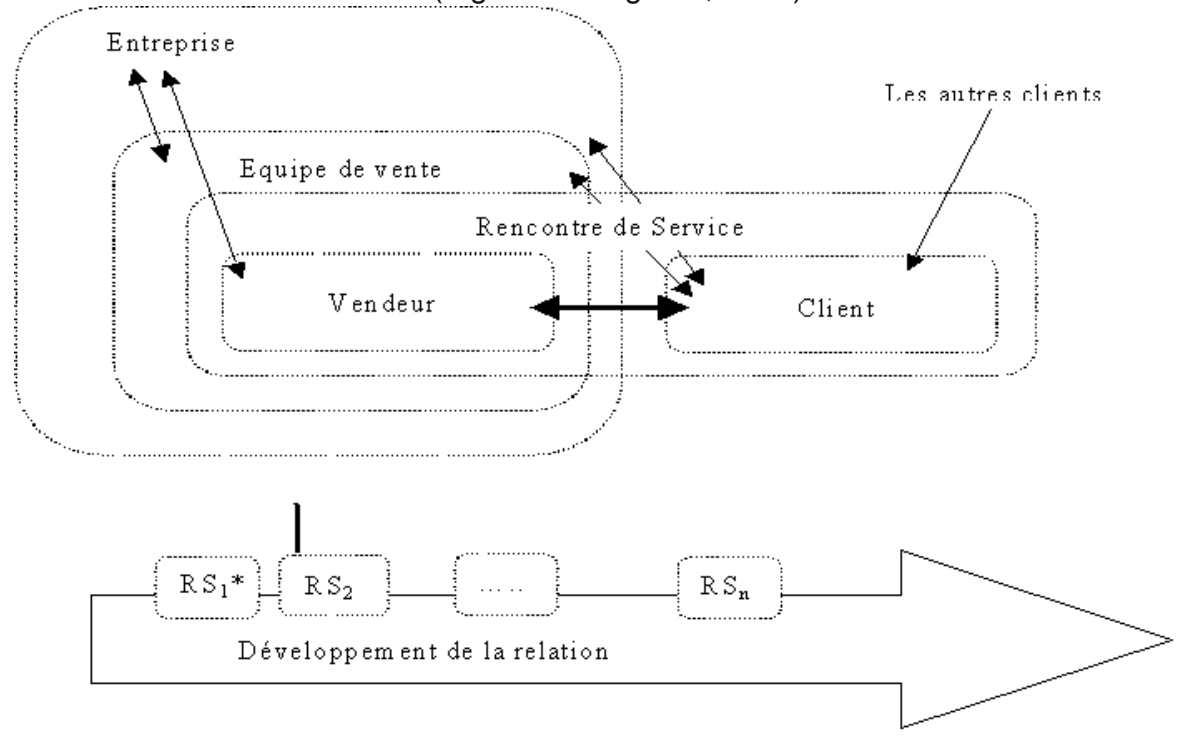

${ }^{*} \mathrm{RS}=$ Rencontre de service

Ce schéma présente les quatre parties prenantes impliquées dans la relation, à savoir : l'entreprise, le vendeur, l'équipe de vente (nous écartons de notre problématique les questions liées au multicanal) et leurs interactions possibles. Nous nous focalisons ici sur l'interaction client-vendeur ou Rencontre de Service (RS).

Des études récentes ont mis en exergue l'influence prédominante des phases d'introduction et de conclusion de la vente sur l'évaluation de la qualité relationnelle (Evans et al, 2000 ; Bergeron et al, 2008). Les impressions du client sont fondées sur les comportements relationnels du vendeur (Bergeron et al, 2008).

Dans le champ du marketing des services, la satisfaction à l'égard du vendeur est ainsi considérée comme un déterminant de la satisfaction globale avant d'être envisagée comme un facteur de la qualité de la relation (Crosby et al, 1990). La qualité de la relation est ici composée de deux dimensions : (1) la satisfaction à l'égard du vendeur, qui renvoie à la satisfaction du client eu égard au rôle et à la performance du vendeur; (2) la confiance à l'égard du vendeur. Selon Dorsch et al (1998), qui ont interrogé les clients sur la qualité de la relation, c'est l'orientation client qui émerge comme la clé de voûte de toute relation. 


\subsection{L'orientation client du vendeur : présentation et limites}

L'orientation client du vendeur s'intéresse aux comportements du vendeur qui lui permettent ne pas considérer son seul intérêt à court terme mais également l'intérêt du consommateur. Intérêts du vendeur et du consommateur ont souvent des temporalités différentes (Kelley, 1992). Selon Saxe et Weitz (1982), 7 comportements délimitent le concept d'orientation client : (1) le désir d'aider le client à prendre de bonnes décisions d'achat ; (2) l'aide au client pour évaluer ses besoins ; (3) l'offre de produits qui satisfont ces besoins ; (4) la description conforme du produit ; (5) l'adaptation des présentations de l'offre à l'intérêt du client ; (6) l'évitement de tactiques d'influence manipulatrices ; (7) l'abstention de l'usage de pression sur le client.

Cependant, l'examen de la littérature laisse émerger des concepts connexes qui sont recouverts par celui de l'orientation client ou qui le dépassent. Ainsi, certains comportements relationnels tels que l'adaptabilité (Weitz et al, 1986), I'orientation long terme (Schultz et Good, 2000), se rapprochent de l'orientation client (Poujol, 2008). Par contre, l'orientation client semble ignorer des comportements relationnels néanmoins importants tels que le processus interpersonnel (Humphreys et Williams, 1996), et notamment, la créativité, la réactivité et la disponibilité du vendeur. Par ailleurs, les comportements du vendeur s'insèrent dans une dynamique de l'échange : si l'orientation client en rend difficilement compte, l'orientation service (Keillor et al, 1999) avec la notion d'aide au client après la vente ou encore l'écoute (Ramsey et Sohi, 1997) se focalisent sur cet aspect (Poujol, 2008).

L'identification des différents comportements relationnels ainsi que l'étude de leur impact sur l'évaluation du service et de la qualité de la relation client ont constitué les deux principaux axes des recherches antérieures. La notion d'orientation client ne semble pas complètement englober l'ensemble des comportements relationnels clés (réactivité, écoute...) aussi nous semble-t-il fondamental d'examiner plus en détail l'orientation client telle qu'elle est perçue par le client de nos jours.

\section{Méthodologie}

Pour comprendre de manière approfondie la notion d'orientation client du vendeur du point de vue des consommateurs, nous avons eu recours à la méthode des incidents critiques qui a pour particularité de s'appuyer sur l'étude des comportements. Après avoir présenté cette méthodologie et sa pertinence, les phases de collecte et de traitement des données sont exposées. 


\subsection{La méthode des incidents critiques}

Flanagan (1954) définit la méthode des incidents critiques (MIC) comme " un ensemble de procédures pour recueillir des observations directes de comportement humain de manière à faciliter leur utilité potentielle dans la résolution de problèmes pratiques et le développement de larges principes psychologiques ». Ross (2002) a identifié deux caractéristiques pour déterminer le caractère critique d'un incident : la perception du répondant et l'aspect conatif de l'événement. En effet, ce dernier affecte le comportement de l'individu et de la relation établie et c'est ainsi que cette méthode est adaptée à notre objectif de recherche.

Cette méthode se révèle particulièrement intéressante pour étudier les attributs pertinents aux yeux du consommateur et pour explorer un sujet qui nécessite une compréhension approfondie (Bitner et al, 1990) ; ce qui correspond à nos objectifs principaux. Cette méthode a permis de classifier les sources de satisfaction et d'insatisfaction dans le domaine des restaurants, hôtels, compagnies aériennes (Bitner, 1990), de comprendre l'impact des autres clients sur l'expérience de service (Grove et Fisk, 1997), les comportements de plainte (Mack et al, 2000) et ceux de changement de fournisseur (Keaveney, 1995). Pratiquement, la méthode des incidents critiques consiste à demander aux répondants de raconter une histoire qui leur est arrivée. II s'agit ainsi d'un exercice de description.

Cette méthode présente deux avantages majeurs. Premièrement, elle génère des descriptions de processus tels que les individus les ont perçus et l'exercice peut être comparable à certaines conversations quotidiennes échangées entre amis ou connaissances. Pour ce faire, les répondants utilisent leurs propres mots et leur propre logique. Deuxièmement, lors de la phase de traitement des données, le travail d'inférence et de conceptualisation revient à l'interviewer (Bitner et al, 1990).

La méthode des incidents critiques s'inscrit donc dans les mesures verbales post exposition et s'appuie sur la description d'un événement perçu, mémorisé et raconté (et par conséquent, interprété) par le répondant.

\subsection{La collecte des données}

La collecte des données a été réalisée au travers d'un formulaire : cette grille d'entretien a permis de recueillir les comportements saillants du vendeur aux yeux des consommateurs sans aucune forme de relance ou d'orientation de la part du chercheur. Ce mode de collecte offre l'opportunité d'un temps de réflexion au répondant pour déterminer l'incident le plus représentatif. De plus, nous avons posé des questions simples et directes à la deuxième personne du pluriel qui facilite l'implication du répondant et renforce la dimension projective de l'enquête. 
Enfin, nous avons adopté une évocation large de la transaction commerciale. Notre objectif est d'étudier l'orientation client des vendeurs dans toute expérience de consommation, le répondant était libre de parler de l'expérience d'achat de son choix.

Le formulaire est composé de trois grandes parties. La première présente l'étude et ses objectifs. Selon les instructions données, les individus doivent ensuite narrer un évènement dont ils ont souvenance, sans a priori sur le dénouement de l'histoire, ni sur le domaine de consommation évoqué. Le formulaire se termine par les caractéristiques sociodémographiques des répondants collectées à l'aide de questions fermées (annexe 1).

Encadré 1 : Présentation de l'enquête

Vous souvenez-vous d'un événement qui vous serait survenu lors de l'achat d'un produit ou d'un service et qui faisait intervenir un vendeur de l'entreprise.

L'issue de cet événement a pu être bénéfique ou non ; ce souvenir peut vous être plaisant ou déplaisant.

Tout d'abord, précisez le service ou le produit dont vous allez parler.

Décrivez de manière précise les circonstances de cet événement. Comment s'est-il déroulé ? Qui étai(en)t présent(s) ? Y avait-il d'autres clients ? Où était-ce ? Pour quelles raisons étiez-vous là ? Aviez-vous déjà consommé ce service ou ce produit?

Qu'avez-vous dit ou fait? Qu'a dit ou fait le vendeur ? Étiez-vous déçu(e) ? Pourquoi ? Quels détails sont restés mémorables pour vous ?....

8

Indiquez également quand cet événement a eu lieu ?

8

\subsection{La description de l'échantillon}

Les incidents critiques ont été collectés auprès d'un échantillon de convenance (étudiants en apprentissage en gestion mais non spécialistes de la vente). 77 formulaires ont été récoltés et analysés. Nous avons écarté dix formulaires pour deux raisons: d'une part, l'incomplétude et la pauvreté de la narration (8 formulaires) et d'autre part, l'absence d'un vendeur ou d'un contact humain dans le récit (2 formulaires). L'échantillon est composé de 49 femmes et de 18 hommes, l'âge moyen des répondants est de 25 ans.

Nous trouvons des expériences de consommation qui ont trait à deux grands univers : d'une part, l'univers des technologies de l'information et de la communication (29 incidents soit $43 \%$ ) et d'autre part, l'univers de la mode (20 incidents soit $30 \%$ ). II est intéressant d'observer que la quasi-totalité des évènements font spontanément référence à des expériences d'achat pour lesquelles les répondants sont fortement impliqués. L'implication est un concept 
ancien et essentiel dans l'étude du comportement du consommateur car elle est " conçue comme un état de motivation, d'excitation et d'intérêt, susceptible de jouer un rôle médiateur important sur le comportement » (Valette-Florence, 1989, p.75). Cet état non observable a une influence sur les différentes phases du comportement du consommateur, notamment le processus de recherche et le traitement de l'information ainsi que la prise de décision.

\subsection{La codification}

Les conclusions de notre étude reposent sur la description et l'interprétation des catégories mises en évidence par l'évaluation qualitative des juges.

Afin d'obtenir la meilleure qualité de codage, nous avons fait appel à trois juges. L'unité d'analyse retenue a été l'histoire narrée sans possibilité de la rendre sécable. Deux juges ont eu pour mission d'établir la grille de codification des données. Ils ont travaillé séparément et ensemble de manière séquentielle afin d'établir les catégories. Ce travail se découpe en quatre grandes étapes.

Le premier juge, spécialiste en comportement du consommateur, a établi de manière intuitive les catégories après plusieurs lectures flottantes. Le deuxième juge, spécialiste en management de la force de vente, a codé les données à l'aide de la grille établie qu'il a complétée afin d'obtenir la meilleure description possible des incidents. Les deux premiers juges ont ensuite travaillé ensemble pour aboutir à une seule grille. Ils ont ensuite codé les données séparément. Les points de désaccord ont été maintenus pour la suite du processus. Enfin, le troisième et dernier juge, spécialiste en gestion de la force de vente, a codé les données à l'aide de la grille proposée, sans la modifier.

Dans une étape ultérieure, nous avons estimé le degré d'accord entre les juges pris deux par deux avec deux indicateurs : le pourcentage d'accord et le coefficient de Kappa (Cohen, 1960). D’après les résultats présentés en tableau 2, les scores des différents indicateurs sont satisfaisants.

Tableau 2 : Estimations du degré d'accord entre les juges

\begin{tabular}{|l|c|c|c|}
\hline & Accord entre juges 1 et 2 & Accord entre juges 2 et 3 & Accord entre juges 1 et 3 \\
\hline Pourcentage d'accord & $88,06 \%$ & $61,19 \%$ & $59,70 \%$ \\
\hline Coefficient de Kappa & $87,20 \%$ & $58,75 \%$ & $57,16 \%$ \\
\hline
\end{tabular}

\section{Résultats et discussion}

La restitution de nos résultats s'articule en trois points. Tout d'abord, nous appréhendons la complexité du métier du vendeur et l'hétérogénéité des situations de vente. Puis, à partir de la définition de l'orientation client énoncée par Saxe et Weitz (1982), nous confirmons les différentes facettes de ce concept 
et soulignons l'incomplétude de ce dernier : ainsi, nous proposons une grille de lecture retravaillée de l'orientation relationnelle. Enfin, notre étude suggère les potentialités du recours à la théorie de l'attachement, initialement développée entre l'enfant et la figure d'attachement pour comprendre l'attachement entre consommateur et vendeur (Ainsworth, 1969 ; Bowlby, 1969).

\subsection{Du vendeur aux situations de vente : une mosaïque de situations}

Les instructions initiales font expressément référence à un « vendeur » sans plus de précision, ce flou volontaire devait nous permettre de mieux cerner ce que recouvre le terme de vendeur aux yeux des consommateurs. Par ailleurs, les consignes demandaient de replacer le vendeur dans une situation de vente. Les résultats montrent à quel point la causalité « un bon vendeur conduit à une bonne vente » est contestable.

\subsubsection{Le vendeur : une pluralité de perceptions}

Malgré les instructions initiales, nous pouvons observer la forte hétérogénéité engendrée par le terme vendeur. II a été choisi car il ne fait pas explicitement référence à un statut (VRP, salarié...) ou à une rémunération, mais met l'accent sur l'activité de vente. Les consommateurs ont évoqué aussi bien le vendeur à domicile que le vendeur salarié d'une entreprise. Ils ont également assimilé à des vendeurs, le patron ou le gérant d'une entreprise (par exemple, un bijoutier, un coiffeur, un pizzaïolo..), le personnel d'un centre d'appel... Autrement dit, aux yeux du consommateur, il semble que tout personnel en contact de l'entreprise soit vecteur de l'image de celle-ci et ainsi, considéré comme vendeur. La caractéristique saillante et déterminante n'est donc ni la rémunération, ni le statut... mais bien la position charnière, le fait d'être à l'interface entre le consommateur et l'entreprise.

Par ailleurs, nous pouvons supposer que, la culture de l'entreprise et sa manière de concevoir les relations commerciales influencent les comportements du vendeur tels qu'envisagés dans notre étude. C'est ainsi que l'orientation marché de l'entreprise affecte positivement l'orientation client du vendeur (Siguaw et al, 1994).

\subsubsection{Les caractéristiques des situations de vente}

Notre étude confirme le lien complexe qui existe entre la perception du vendeur et le souvenir de l'expérience de consommation. Nous avons codifié les incidents selon leur caractère satisfaisant ou insatisfaisant et selon la présence ou l'absence de transaction commerciale (tableau 3). A partir de cette codification, nous pouvons émettre quatre principales remarques. 
Premièrement, les consommateurs ont spontanément narré plus d'incidents satisfaisants (39 sur 67) que d'incidents insatisfaisants (28 sur 67). Ils semblent donc plus concernés par leur expérience positive vécue avec un vendeur. Deuxièmement, la grande majorité des incidents inclut une transaction commerciale (57 évènements sur 67). Troisièmement, nous constatons que l'absence d'achat ne correspond pas nécessairement à un souvenir déplaisant et inversement l'achat n'est pas systématique perçu comme un souvenir plaisant. A titre d'exemple, mentionnons les conseils donnés par ce vendeur qui ne conduisent pas à un achat :

Je suis allée chez Darty avec mon conjoint pour acheter une crédence. J'ai demandé le prix à un vendeur d'une crédence exposée sur le mur. II m'a répondu qu'il serait préférable que je l'achète dans un magasin tel que lkea car elle était trop chère !! J'ai été très surprise car d'habitude, les vendeurs de ce magasin font tout pour vous vendre le maximum de produits... J'ai été très étonnée, mais satisfaite car le vendeur avait pris la place d'un conseiller plutôt que d'un pur commercial.

Inversement, l'achat ne traduit pas pour autant un comportement relationnel du vendeur.

Achat d'un lecteur MP3 chez Carrefour. Altercation avec un vendeur du rayon qui voulait absolument me faire acheter un Ipod Shuffle. Je lui explique au moins 5 fois que je ne souhaite pas acheter le produit mais le lecteur Creative. Très déçue par l'attitude négative du vendeur. II ne m'a pas écouté. J'ai fini par prendre le lecteur MP3 que je souhaitais moimême.

Quatrièmement, la relation complexe entre comportements du vendeur et réactions du consommateur nous a conduits à distinguer les évènements narrant une vente de ceux évoquant la simple distribution d'un produit ou d'un service. De fait, nous entendons par «vente » l'ensemble des actions mises en œuvre par le vendeur même si ces derniers ne sont pas concluants. Dans ces situations, le vendeur a écouté le client, compris ses attentes, proposé une prestation correspondante. Dans la distribution, le vendeur ne fait que « donner » le produit ou le service demandé. Dans cette perspective, certains outils tels qu'une meilleure signalétique ou un merchandising plus performant pourraient le remplacer.

Exemple de vente :

[...] Je voulais lui [ami] acheter un costume pour son anniversaire. J'ai regardé, tout d'abord, une chemise, le vendeur est venu et m'a présenté tous les articles du magasin alors que je n'avais rien demandé. Finalement, nous n'avons rien acheté car nous voulions à tout prix nous " débarrasser 》 de ce vendeur beaucoup trop impliqué. 
Exemple de distribution :

C'était à une heure de pointe dans une grande librairie très fréquentée. [...] Il m'a fallu demander à un vendeur à quel étage se trouvait le domaine qui m'intéressait. Celui-ci a répondu d'un air dédaigneux et sur un ton sec après plusieurs demandes. II m'a dit que c'était mal indiqué mais que nous pouvions quand même chercher!

Contrairement à ses collègues, il n'a pas voulu chercher le livre désiré et en conseiller d'autres.

Le croisement des différentes codifications des incidents critiques collectés permet d'associer les expériences de vente à des expériences satisfaisantes alors que les expériences de distribution sont plutôt ressenties comme insatisfaisantes (Tableau 3 ci-dessous). En effet, 30 incidents (soit $45 \%$ ) sont simultanément codifiés comme satisfaisants et retracent des expériences de vente alors que 19 incidents (soit $28 \%$ ) correspondent à des expériences de distribution et sont vécus comme insatisfaisants. Cependant, la faiblesse de notre échantillon ne nous permet de conclure à une relation statistique entre ces deux notions.

Tableau 3 : Satisfaction et relation commerciale (vente / distribution / absence d'achat)

\begin{tabular}{|l|c|c|c|c|}
\hline & \multicolumn{2}{|c|}{ Transaction commerciale } & \multirow{2}{*}{$\begin{array}{c}\text { Absence de } \\
\text { transaction }\end{array}$} & \\
& $\begin{array}{c}\text { Expérience de } \\
\text { vente }\end{array}$ & $\begin{array}{c}\text { Expérience de } \\
\text { distribution }\end{array}$ & & Total \\
\hline Expérience satisfaisante & 30 & 7 & 2 & 39 \\
\hline Expérience insatisfaisante & 1 & 19 & 8 & 28 \\
\hline Total & 31 & 26 & 10 & 67 \\
\hline
\end{tabular}

\subsection{L'enrichissement de l'orientation client : de la théorie à la pratique}

Notre travail inductif (tableaux 4 et 5 ) rejoint les sept facettes de l'orientation client proposées par Saxe et Weitz (1982). Cependant, les deux aspects « évitement de tactiques d'influence manipulatrice " et " abstention de l'usage de pressions sur le client » ont été regroupés sous la dimension éthique. Sans doute, la faiblesse de notre échantillon ne permet pas de mettre en lumière ces dimensions qui nécessiteraient des investigations complémentaires. Par ailleurs, notre analyse fait aussi ressortir des sous-catégories pour certaines facettes. Ainsi, le désir d'aider le client à prendre de bonnes décisions d'achat peut se traduire par deux comportements du vendeur : aider le client à l'évaluation des différentes possibilités et développer un argumentaire commercial. Nous illustrons chaque facette de l'orientation client telle qu'elle est envisagée par Saxe et Weitz (1982) avec les incidents critiques typiques. 
Tableau 4 : Grille de codification

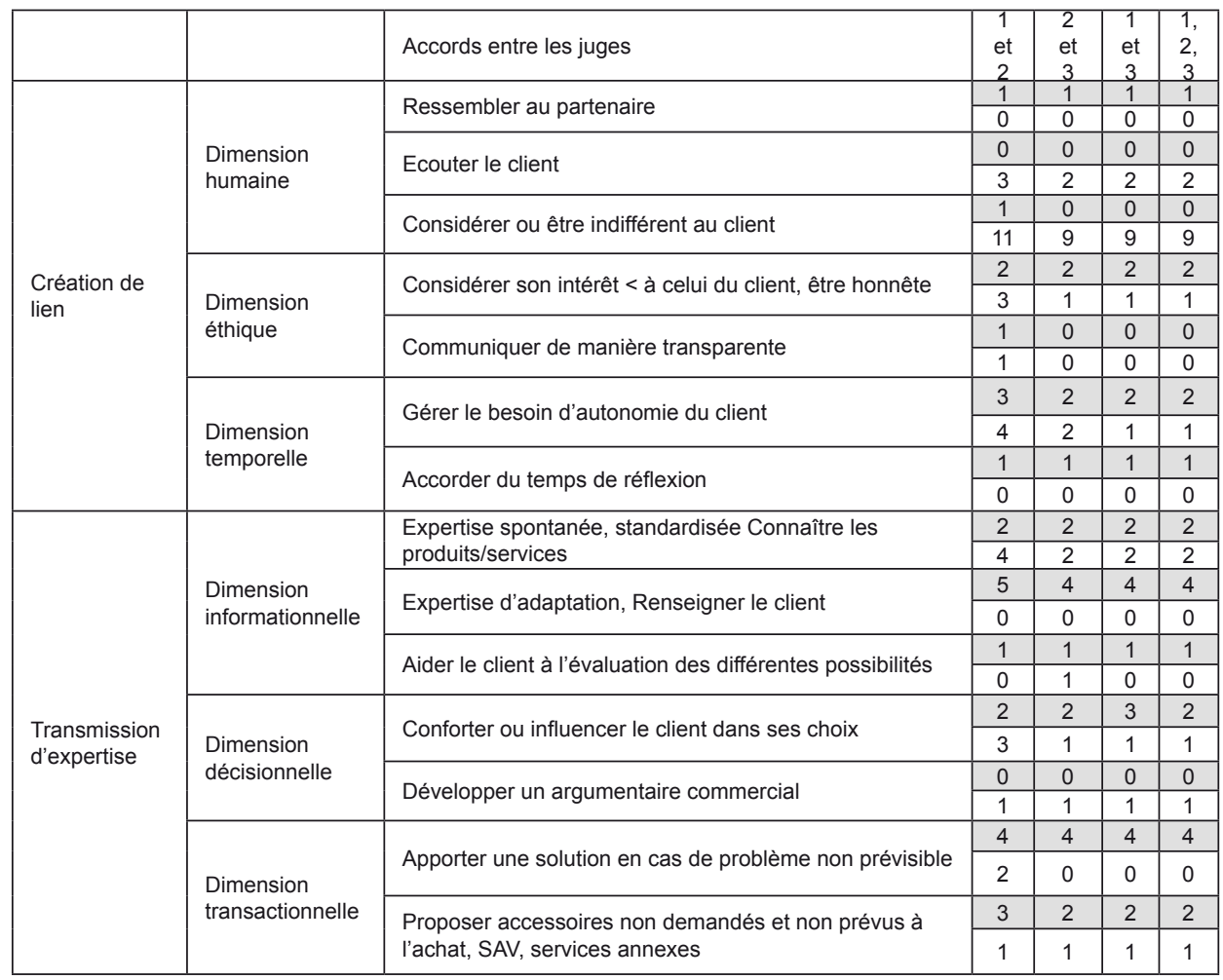

Chaque comportement indiqué par un verbe à l'infinitif a été doublement codifié : dans sa version positive (par exemple, écouter le client : cette codification correspond aux lignes grisées de chaque comportement) et dans sa version négative (par exemple, ne pas écouter le client : ce codage correspond aux lignes blanches de chaque comportement). 
Tableau 5 : Confrontation des dimensions de l'orientation client proposées par Saxe et Weitz (1982) et celles émergeant de notre étude des incidents critiques

\begin{tabular}{|c|c|}
\hline $\begin{array}{l}\text { Facettes de } \\
\text { l'orientation client }\end{array}$ & $\begin{array}{l}\text { Facettes de l'orientation relationnelle mise en perspective par notre codification et } \\
\text { exemples de verbatim. }\end{array}$ \\
\hline $\begin{array}{l}\text { Désir d'aider le } \\
\text { client à prendre de } \\
\text { bonnes décisions } \\
\text { d'achat }\end{array}$ & $\begin{array}{l}\text { Aider le client à l'évaluation des différentes possibilités } \\
\text { Développer un argumentaire commercial } \\
\text { [...] je me suis adressée à un vendeur responsable du rayon. Ayant eu une mauvaise } \\
\text { expérience avec la marque d'ordinateur portable qui paraissait être le plus intéressant, } \\
\text { je me suis permise de lui en faire part. Après discussion, celui-ci m'a rassurée et j'ai } \\
\text { finalement acheté le produit le jour même. Contente de ne pas avoir eu à faire tous les } \\
\text { magasins pour trouver un vendeur capable de me renseigner. }\end{array}$ \\
\hline $\begin{array}{l}\text { Offre de produits } \\
\text { qui satisfont ces } \\
\text { besoins }\end{array}$ & $\begin{array}{l}\text { Expertise d'adaptation, Renseigner le client } \\
\text { C'était à la FNAC [...]. J'étais venue sans avoir une réelle idée du modèle [...] mais je } \\
\text { connaissais mon budget (seule limite pour l'achat). Je suis allée voir le premier vendeur } \\
\text { que j'ai trouvé et par chance, il était bon connaisseur et il a su me proposer un bon } \\
\text { produit tout en respectant mon budget. }\end{array}$ \\
\hline $\begin{array}{l}\text { Aide au client } \\
\text { pour évaluer ses } \\
\text { besoins }\end{array}$ & $\begin{array}{l}\text { Conforter ou influencer le client dans ses choix } \\
\text { Arrivée dans la boutique, j'ai repéré le portant puis retrouvé ma taille et ensuite essayage } \\
\text { en cabine. [...]. Pas de déception, au contraire, elle [vendeuse] m'a confortée dans mon } \\
\text { choix et l'association faite. }\end{array}$ \\
\hline $\begin{array}{l}\text { Description } \\
\text { conforme du } \\
\text { produit }\end{array}$ & $\begin{array}{l}\text { Expertise spontanée, standardisée Connaître les produits/services } \\
\text { Je suis allée chez un revendeur de matériel informatique. [...] Je voulais des } \\
\text { renseignements sur les différentes configurations et surtout avoir un avis concernant } \\
\text { les différents constructeurs. Le vendeur avait l'air de bien s'y connaître et avait le mérite } \\
\text { d'être franc (du moins c'est l'impression qu'il me donnait). }\end{array}$ \\
\hline $\begin{array}{l}\text { Adaptation des } \\
\text { présentations de } \\
\text { l'offre à l'intérêt du } \\
\quad \text { client }\end{array}$ & $\begin{array}{l}\text { Apporter une solution en cas de problème non prévisible } \\
\text { Proposer accessoires non demandés et non prévus à l'achat, SAV, services } \\
\text { annexes } \\
\text { J'étais à la recherche d'un ordinateur portable [...] il [vendeur] a confirmé mon choix par } \\
\text { ses conseils, mais malheureusement, il ne restait plus de ce modèle en magasin. Donc, } \\
\text { il m'a proposé d'acheter le modèle en exposition avec une réduction commerciale de } \\
5 \% \text {. Donc, je l'ai acheté et je suis repartie satisfaite. }\end{array}$ \\
\hline $\begin{array}{c}\text { Evitement } \\
\text { de tactiques } \\
\text { d'influence } \\
\text { manipulatrice } \\
\text { Abstention } \\
\text { de l'usage de } \\
\text { pressions sur le } \\
\text { client }\end{array}$ & $\begin{array}{l}\text { Dimension éthique } \\
\text { D'un coup, un des deux vendeurs s'est jeté sur moi pour me fourrer deux jeans qui } \\
\text { " d'après lui » serait « pour moi », mais en réalité pas à mon goût. II m'a entraîné dans la } \\
\text { cabine d'essayage, les jeans sous le bras et m'a laissé me changer tout en m'attendant } \\
\text { de l'autre côté du paravent. Je suis sortie et là, il m'a quasiment imposé d'acheter un des } \\
\text { jeans. [...] Je me suis demandée s'il faisait toujours ça et s'il y a des gens qui acceptent } \\
\text { d'acheter quelque chose car on le leur « impose ». }\end{array}$ \\
\hline
\end{tabular}

Conformément à la littérature (Saxe et Weitz, 1982 ; Biong et Selnes, 1996; Humphreys et Williams, 1996), un « bon » vendeur est, par conséquent, celui qui maîtrise et fournit les caractéristiques techniques des produits, propose des services annexes ou des accessoires. Cette expertise se révèle cruciale à trois moments clés de l'échange mis en évidence dans les modèles de comportement du consommateur : la collecte d'informations, la prise de décision, l'exécution de la transaction. Le vendeur intervient à chaque phase comme un expert, en apportant son savoir. Néanmoins, le corpus des histoires nous conduit également à élargir les facettes de l'orientation relationnelle du vendeur. 
A côté de la dimension technique du vendeur, que nous avons appelée "la transmission d'expertise », le client est également sensible à la relation que le vendeur établit avec lui. La " bonne » relation avec un vendeur peut se décrire comme un lien qui unit les deux. Selon notre analyse, cette création de lien se décompose en trois dimensions dont 2 inédites : la dimension éthique mise en exergue dans les travaux antérieurs (tableau 4), la dimension humaine et la dimension temporelle.

La dimension humaine, le fait que le vendeur considère ou soit indifférent à son client, apparaît essentielle dans le discours des clients. Ceci montre l'importance d'être connu et reconnu comme être humain dans la relation commerciale. A ce titre, notons que c'est souvent l'absence de marques de politesse ou d'attentions (9 incidents sur 67) qui est relevé par les clients ou l'impression désagréable d'être transparent.

Je suis allée acheter une pizza chez le " pizzallologue » [...]. Il y avait trois clients et une vendeuse. [..] Je suis entrée et j'ai dit « bonsoir ». La vendeuse (gérante de la pizzeria) n'ayant pas entendu m'a répété trois fois bonsoir sur un ton agressif. Elle m'a ensuite demandé ce que je voulais. Puis, a continué à être agressive. Très déçue de l'accueil, nous sommes parties sans pizza.

Notre troisième sous-catégorie de la création de lien fait référence aux aspects temporels de la transaction. Cet aspect est, à notre connaissance, peu étudié dans la littérature. A la lecture des incidents critiques, le "bon" vendeur est celui qui " gère le besoin d'autonomie du client ». Ceci peut se traduire par deux comportements : aller au devant du client immédiatement en lui proposant de l'aide, ou, au contraire, proposer son aide au moment opportun. Ainsi, la diligence du vendeur peut être difficilement vécue par certains clients qui souhaitent un temps de découverte personnel ou une autonomie. Aucune caractéristique des consommateurs qui peut expliquer ce comportement n'a pu être mise en évidence et il semble difficile de le discerner a priori. Le client apprécie l'attention que lui porte le commercial pour l'aider, mais il est sensible à la manière ainsi qu'au moment où cette aide est proposée. II est intéressant de voir comment les efforts des vendeurs sont parfois inutiles ou perçus comme une véritable agression comme l'indiquent les dires de ces répondants :

[...] dans le magasin Camaïeu. J'y suis allée pour regarder les nouveaux produits [...]. J'y ai apprécié la responsable du magasin qui a laissé libre cours à mon passage dans le magasin. Je lui ai ensuite demandé si elle avait une jupe. J'ai aimé sa manière de me conseiller sans vouloir absolument que j'essaie les vêtements. Une relation plus informelle que formelle s'est créée.

Achat d'un meuble. [...]. Le vendeur a insisté pour me conseiller alors que je préférais décider seule, malgré mes refus, il est revenu plusieurs fois à 
la charge pour que j'achète à tout prix. Le vendeur était " trop lourd". Je me suis forcée à rester polie.

La dimension temporelle avec ses deux sous-catégories (gérer le besoin d'autonomie du client et accorder du temps de réflexion) souligne le besoin du client d'effectuer des allers-retours entre la certitude (sécurité) que représente le vendeur, et l'incertitude (doute) que représente la découverte en solitaire. Ces allers-retours sont nécessaires à l'autonomie et l'individualisation des décisions du client.

Dans une perspective constructiviste, Bergadaà et Coraux (2008) soulignent le rôle support du vendeur dans la réassurance du client dans la décision d'achat et la co-gestion du risque d'achat. Le lien vendeur-client semble fondamental dans le processus d'achat et la théorie de l'attachement utile pour mieux comprendre la création de ce lien.

\subsection{Les perspectives offertes par la théorie de l'attachement}

Le lien vendeur-client est caractérisé par sa dimension temporelle, l'importance d'une co-construction (allers-retours entre vendeurs et consommateurs) et le respect de l'être humain et de sa personnalité. Ainsi, il est intéressant d'étudier cette relation en mobilisant la littérature sur la construction du lien originel qu'aborde la psychologie génétique et notamment la théorie de l'attachement. Après la présentation des principaux fondements de la théorie de l'attachement, nous établissons des ponts entre notre étude et cette théorie.

\subsubsection{Principaux fondements de la théorie de l'attachement}

Le processus de l'attachement de l'enfant à une figure d'attachement (Ainsworth, 1969 ; Bowlby, 1969) correspond à un processus indispensable d'échange pour la survie et l'évolution de l'enfant au sein de la société. Celui-ci s'avère prédictif du développement ultérieur des rapports au monde en construisant des liens sécurisants, anxieux ou ambivalents.

En schématisant, la construction de l'attachement est constituée d'une première étape avec la figure d'attachement qui consiste à une reconnaissance réciproque des deux acteurs (la mère bien souvent et l'enfant). Ce sont les échanges comportementaux et sensoriels ainsi que le discours de la figure d'attachement à l'enfant, additionnés d'un phénomène physiologique de " bonding », qui explique l'établissement du lien chez l'homme (Pierrehumbert et al, 2002). Cette première accroche mutuelle sollicite l'émotion comme élément d'échange et de premier rapport au monde. La figure d'attachement présente l'enfant au monde et le monde à l'enfant, devenant valeur de référence pour ce dernier (Pierrehumbert, 2003). Chez l'enfant, cette étape de protection conditionne la nature du lien créé : sécurisé ou anxiogène. La figure d'attachement incite l'enfant à s'intéresser au 
monde extérieur par lequel il est attiré mais aussi angoissé (Winnicott, 1989). Cette phase de " co-construction » et de " co-traduction » du monde extérieur participe à l'acquisition de l'autonomie de l'enfant vis-à-vis de la référence originelle qu'est la figure d'attachement. Les allers-retours entre l'exploration extérieure et la réassurance régressive permettent, notamment par la médiation du langage, de proposer un nouvel individu adapté au monde qui l'entoure lui permettant de structurer son intelligence d'adaptation (Piaget, 1950). Les deux personnes tendent à s'individualiser devenant deux personnalités différenciées d'autant mieux que la figure d'attachement reste positivement présente dans la vie de l'enfant : c'est la persistance de l'objet d'attachement (Zazzo, 1974). Les deux personnes peuvent alors s'individualiser puis se revoir pour des échanges privilégiés.

Winefield et al (1989) ont examiné la persistance de l'effet de la qualité de la relation élaborée dans l'enfance à l'adaptation positive de l'adulte dans son environnement. Ainsi, les stratégies d'attachement, une fois construites ont tendance à se reproduire à l'âge adulte comme en attestent les études de la psychologie du développement (Flaherty et Richman, 1986; BakermansKranenburg et ljzendoorn, 1993). Par ailleurs, les travaux sur la relation au monde à l'âge adulte ont mis en évidence cette classification dans le contexte de l'attachement amoureux (Hazan et Shaver, 1987) et de l'attachement à la marque (Thomson et al, 2005).

\subsubsection{Résultats de notre étude vus sous l'angle de la théorie de l'attachement}

Nous pouvons établir une comparaison entre l'acte de vente (relation vendeurclient) et l'attachement (relation mère-enfant). Trois éléments sont à souligner : l'importance du " premier » contact ; la phase de co-construction et la capacité relationnelle du vendeur au travers notamment de l'empathie.

Dans notre étude, le premier contact et les premiers moments de la rencontre vendeur-client semblent décisifs. Le vendeur doit établir d'emblée un bon contact avec le client. Ceux-ci sont particulièrement marqués par ces échanges, moment où les deux individus se rencontrent pour s'identifier, se connaître et se mettre à la portée l'un de l'autre pour communiquer au mieux. Cette phase repose sur des perceptions sensorielles, notamment, la vue donnant lieu à des émotions. Pour Bagozzi et al (1999), une émotion est un état mental soudain faisant suite à des évaluations cognitives d'événements ou de pensées. Elle s'accompagne d'un processus physiologique souvent exprimé physiquement (gestes, expressions faciales) et qui peuvent se traduire par des comportements.

Deuxièmement, l'objet est mentionné comme l'élément autour duquel se construit la transaction entre une personne et son environnement relationnel et non pour sa seule valeur intrinsèque. A l'image du rôle rassurant du discours de la mère, 
le discours du vendeur remplirait une fonction similaire d'enracinement du lien permettant à son client d'intégrer des expériences de consommation non vécues jusqu'alors. Ainsi, la valeur ajoutée du vendeur, créateur de lien, consisterait à présenter le produit ou le service comme un élément nouveau s'intégrant dans l'environnement du client. En effet, cet élément est vécu comme source d'angoisse ou comme une solution en fonction de l'implication du vendeur. Le rôle du vendeur se situerait donc autour de la réassurance qu'il apporte à son client en adaptant la solution produit à l'environnement de ce dernier. Goudarzi et Eiglier (2006) évoquent des phénomènes assez proches pour des activités de service en soulignant l'activité d'apprentissage réciproque qui participe à la socialisation organisationnelle des clients.

Enfin, les incidents critiques répertoriés dans la catégorie "création de lien » illustrent la notion d'empathie au travers de ses dimensions comportementales, sociales et psychologiques (Berthoz et Jorland, 2004). C'est en effet, au cours de la phase de découverte des besoins du client et de celle de l'élaboration d'un argumentaire commercial, que le vendeur développe des compétences d'empathie lui permettant d'adhérer au monde de l'autre sans perdre sa propre identité. Autrement dit, le vendeur doit comprendre le monde de son client sans y adhérer entièrement ce qui serait la preuve de sa sympathie (Fonagy et al, 1993). L'empathie permet au vendeur de proposer une solution appropriée à l'acheteur tout en le guidant dans sa décision d'achat.

\section{Apports, limites et voies futures de recherche}

Dans la perspective de mieux comprendre le rôle des vendeurs dans la construction de relations commerciales, en articulant les niveaux entreprise-vendeur-client, nous nous sommes focalisés sur l'interaction commerciale.

Du point de vue théorique, nous avons revisité un concept largement étudié dans la littérature au cours des années 80 : l'orientation client du vendeur. Des apports théoriques récents ont montré que d'autres aspects relationnels sont importants dans la vente. De fait, nous avons voulu mieux comprendre ce qui comptait pour le client dans son interaction avec un vendeur. La théorie de l'attachement est apparue comme un cadre théorique pertinent pour analyser la relation vendeurclient et souligner, en particulier, la phase de création du lien.

D'un point de vue managérial, une meilleure compréhension de la construction du lien entre le vendeur et le client permet de mettre à jour trois voies d'amélioration de la gestion de la qualité de la relation client: (1) la prise de contact est une étape qui passe essentiellement par le langage corporel et l'apprivoisement temporel des deux parties. La gestion des distances, la mise en recul ainsi que la compréhension de la disponibilité voulue par l'autre sont autant de compétences que doit avoir le vendeur; (2) la découverte des besoins et l'argumentation 
commerciale demandent au vendeur de passer constamment de son univers (notamment, en termes de formation technique) à l'univers du client (notamment en termes d'utilisation). L'empathie c'est-à-dire la capacité à " se décentrer » est une compétence fondamentale et indispensable ; (3) la conclusion de la vente nécessite, pour le vendeur, de savoir identifier la nature du lien d'attachement et d'imaginer un futur possible ainsi que les actions inhérentes.

L'identification des ces compétences clés aura des répercussions indéniables sur les recrutements et la formation des commerciaux et des managers mais aussi sur la gestion des lieux de vente (magasins en particuliers) dans lesquels les vendeurs participeraient davantage à une mise en scène des produits pour accompagner le client dans sa vente.

Nos résultats ont été établis sur la base d'un échantillon modeste (67) d'observations à l'aide d'une méthode spécifique, ce qui limite certainement la généralisation de nos résultats. Une étude quantitative permettrait de pallier la première limite de ce travail. Une deuxième limite a trait à la collecte des données qui a fait appel à la mémoire. Se souvenir est une activité soumise aux filtres individuels. Malgré les précautions prises nos résultats sont à prendre avec prudence. Ils pourraient s'enrichir d'une confrontation avec les travaux sur les émotions.

Les incidents qui traitent de la transmission d'expertise, plus largement positifs, soulignent les compétences des vendeurs alors que les incidents relatifs à l'axe " création de lien » mentionnent davantage des manquements du vendeur. Audelà d'un biais lié à la collecte des données, il serait intéressant d'investiguer cette particularité : De l'incompétence des vendeurs dans une optique relationnelle? Est-ce le signe de l'évolution d'un métier?

Les relations complexes et ambiguës entre l'acte d'achat (que celui-ci corresponde à de la vente ou de la distribution) et la satisfaction de l'expérience vécue constituent une voie intéressante de recherche. Existe-t-il une structure interne théorique reliant ces différents concepts ? Les relations causales entre d'une part, les composantes de l'orientation relationnelle (transmission d'expertise, création de lien) et d'autre part, les concepts de qualité relationnelle (satisfaction, confiance, engagement vis-à-vis du vendeur et vis-à-vis de l'entreprise) apparaissent également comme une voie de recherche prometteuse. Une autre voie de recherche consiste à étudier le niveau de participation du client à la prestation de service ainsi que le rôle modérateur des caractéristiques individuelles. Par exemple, la théorie de l'attachement suggère que la figure d'attachement prépondérante reste la mère dans nos sociétés occidentales. Pouvons-nous en conclure que les femmes sont par nature plus attentives au lien que les hommes ? Si les étapes de début et de fin de process de vente ont une influence importante, le contenu des étapes intermédiaires qui font appel à 
la confiance méritent d'être investiguées à la lumière de la construction du lien au fil du temps.

\section{Bibliographie}

Ainsworth M. (1969), "Object relations, dependency, and attachment: a theoretical review of the infant-mother relationship", Child Development, Vol.40, n4, p.969-1025.

Bakermans-Kranenburg M.J. et Van Ijzendorroorn M. (1993), "A psychometric study of the adult attachment interview: reliability and discriminant validity”, Developmental Psychology, Vol.29, n5, p.870-879

BaldaufA. et Cravens D.W. (1999), "Improving the effectiveness of field sales organizations: An European perspective", Industrial Marketing Management, Vol.28, n¹, p.63-72.

Bergadaà M. et Bernard J. (1998), "La révolution vente », Décisions Marketing, Vol.13, p.37-45.

Bergadaà M. et Coraux G. (2008), « Le risque d'achat : confrontation des perspectives du client et du commercial », Management et Avenir, Vol.16, p.183-203.

Bergeron J. Fallu J.M. et Roy J. (2008), « Une comparaison des effets de la première et de la dernière impression dans une rencontre de vente ", Recherche et Applications en Marketing, Vol.23, $\mathrm{n}^{\circ} 2$, p.19-36.

Berthoz A. et Jorland G. (2004), L'empathie, Paris, Odile Jacob.

Bettencourt L.A. et Brown S.W. (1997), "Contact employees: Relationships among workplace fairness, job satisfaction and prosocial service behaviours", Journal of Retailing, Vol.73, $n^{\circ} 1, p .39-61$.

Biong H. et Selnes F. (1996), "The strategic role of the salesperson in established buyerseller relationships", Journal of Business to Business Marketing, Vol.3, n³, p.39-78.

Bitner M. J. Booms B. H. et Tetreault M.S. (1990), "The service encounter: Diagnosing favorable and unfavorable incidents", Journal of Marketing, Vol.54, n¹, p.71-84.

Bowlby J. (1969), « Attachement et perte », Paris, PUF.

Cohen J. (1960), "A coefficient of agreement for nominal scales", Educational and Psychological Measurement, Vol.20, p.37-46.

Crosby L.A. Evans K.R. et Cowles D. (1990), "Relationship quality in services selling an interpersonal influence perspective”, Journal of Marketing, Vol.54, $n^{\circ} 3, p .68-81$.

Dorsch M.J. Swanson S.M. et Kelley S.W. (1998), "The role of relationship quality in the stratification of vendors as perceived by customers", Journal of the Academy of Marketing Science, Vol.26, n², p.128-142.

Dwyer R.F. Schurr P.H. et Oh S. (1987), "Developing buyer-seller relationships”, Journal of Marketing, Vol.51, $\mathrm{n}^{\circ} 2$, p.11-27.

Eiglier P. et Langeard E. (1987), Servuction, le marketing des services, Paris, Collection Stratégie et Management, Ediscience International.

Evans K.R. Kleine III R.E. Landry T.D. et Crosby L.A. (2000), "How first impressions of a customer impact effectiveness in an initial sales encounter", Journal of the Academy of Marketing Science, Vol.28, $\mathrm{n}^{\circ} 4, \mathrm{p} .512-526$.

Flaherty J. et Richman J. (1986), "Effects of childhood relationships on the adult's capacity to form social supports", American Journal of Psychiatry, Vol.143, n7, p.851-855.

Flanagan J C. (1954), "The critical incident technique", Psychological Bulletin, Vol.51, n4, p.327-358.

Fonagy P. Steele M. Moran G.S. et Steele H. (1993), "Measuring the ghost in the nursery: An empirical study of the relation between parents' mental representations of childhood experiences and their infants' security of attachment", Journal of the American Psychoanalytic Association, Vol.41, n4, p.957-989

Garbarino E. et Johnson M.S. (1999), "The different roles of satisfaction, trust, and 
commitment in customer relationships”, Journal of Marketing, Vol.63, n², p.70-87.

Goff G. Boles J.S. Bellenger D.N. et Stojack C. (1997), "The influence of salesperson selling behaviours on customer satisfaction with products", Journal of Retailing, Vol.73, $\mathrm{n}^{\circ} 2$, p.171-184.

Goudarzi K. Eiglier P. (2006), "La socialisation organisationnelle du client dans les entreprises de service : concept et dimensions ", Recherche et Applications en Marketing, Vol.21, n³, p.65-90.

Grönroos C. (1990), "Relationship approach to marketing in service contexts : The marketing and organizational behavior interface", Journal of Business Research, Vol.20, $\mathrm{n}^{\circ} 1, \mathrm{p} .3-11$.

Grove S.J. et Fisk R.P. (1997), "The impact of other customers on services experiences : A critical incident examination of "getting along"'”, Journal of Retailing, Vol.73, $\mathrm{n}^{\circ} 1, \mathrm{p} .63-$ 85.

Hazan C. et Shaver P. (1987), "Romantic love conceptualized as an attachment process", Journal of Personnality and Social Psychology, Vol.52, n³, p.511-524

Humphreys M.A. et Williams M.R. (1996), "Exploring the relative effects of salesperson interpersonal process attributes and technical product attributes on customer satisfaction", Journal of Personal Selling and Sales Management, Vol.16, n³, 47-57.

Jaworski B.J. et Kohli, A.K. (1993), "Market orientation: antecedents and consequences", Journal of Marketing, Vol.57, n³, p.53-70.

Keaveney S.M. (1995), "Customer switching behaviour in service industries: An exploratory study", Journal of Marketing, V59, n², p.71-82.

Keillor B.D. Parker S.R. et Pettijohn C.E. (1999), "Sales force performance and aspects of relational selling: implications for sales managers", Journal of Marketing Theory and Practice, Vol.7, n¹, p.101-116.

Kelley S.W. (1992), "Developing customer orientation among service employees", Journal of the Academy of Marketing Science, Vol.20, n¹, p.27-36.

Mack R. Muller R. Crotts J. et BroderickA. (2000), "Perceptions, corrections and defections: Implications for service recovery in the restaurant industry", Managing Service Quality, Vol.10, n6, p.339-346.

Moncrief W.C. et Marshall G.W. (2005), "The evolution of the seven steps of selling", Industrial Marketing Management, Vol.34, $n^{\circ} 1$, p.13-22.

Moulins J-L. (1998), « Etat de fidélité et relation de fidélité : Eléments de réflexion pour une nouvelle approche de l'échange ", Décisions Marketing, Vol.3, janvier-avril, p.67-73.

Morgan R.M et Hunt S.D. (1994), "The commitment-trust theory of relationship marketing", Journal of Marketing, Vol.58, n³, p.20-38.

Piaget J. (1950), « La causalité physique chez l'enfant », Paris, PUF.

Pierrehumbert B. (2003), "Le premier lien : théorie de l'attachement », Paris, Odile Jacob.

Pierrehumbert B. Bader M. Mazet PH. Amar M. et Harlfon O. (2002), Traumatisme dans l'enfance, exclusion défensive et conduites de dépendance à l'adolescence, dans "Sens et non sens de la violence ", Paris, PUF.

Podsakoff P.M. et MacKenzie S.B. (1997), "Impact of organizational citizenship behavior on organizational performance: A review and suggestion for future research", Human Performance, Vol.10, $n^{\circ} 2$, p.133-142.

Poujol F. (2008), "Le rôle des comportements relationnels des commerciaux dans la gestion de la relation client ", Revue Management et Avenir, Vol.16, p.120-138.

Ramsey R.P. et Sohi R.S. (1997), "Listening to your customers: The impact of perceived salesperson listening behavior on relationship outcomes", Journal of the Academy and Marketing Science, Vol.25, $\mathrm{n}^{\circ} 2, \mathrm{p} .127-138$.

Ross J. (2002), "Method of investigating critical incidents: A comparative review", Journal of Service Research, Vol.4, n³, p.193-204. 
Saxe R. et Weitz B.A. (1982), "The S.O.C.O scale: A measure of customer orientation of salespeople", Journal of Marketing Research, Vol.19, n³, p.343-351.

Schultz R.J. et Good D. J, (2000), "Impact of the consideration of future sales consequences and customer-oriented selling on long-term buyer-seller relationships", Journal of Business and Industrial Marketing, Vol.15, n4/5, p.200-215.

Siguaw, J.A. et Brown, G, (1994), "The influence of the market orientation of the firm on sales force behavior and attitude", Journal of Marketing Research, Vol.31, n¹, p.106116.

Tanner J.F. Jr. Fournier C. Wise A. Hollet S. et Poujol J. (2008), “Executives' perspectives of the changing role of the sales profession: understanding their vision of the future", Journal of Business and Industrial Marketing, Vol.23, n³, p.193-202.

Thomson M. Maclnnis J. D. et Whan Park C. (2005), « Les liens attachants : mesurer la force de l'attachement émotionnel des consommateurs à la marque ", Recherche et Applications en Marketing, Vol.20, $n^{\circ} 1,79-98$.

Valette-Florence P. (1989), « Conceptualisation et mesure de l'implication », Recherche et Applications en Marketing, Vol.4, $n^{\circ} 1$, p.57-78.

Weitz B.A. Sujan H. et Sujan M. (1986), "Knowledge, Motivation, and Adaptive Behavior: A Framework for Improving Selling Effectiveness", Journal of Marketing, Vol.50, $n^{\circ} 4$, p.174191.

Williams M.R. (1998), “The influence of salespersons' customer orientation on buyerseller relationship development", Journal of Business and Industrial Marketing, Vol.13, $\mathrm{n}^{\circ} 3$, p.271-287.

Winefield H. Goldney R. Tiggemann M. et Winefield A. (1990), "Parental rearing behaviours: Stability of reports over time and relation to adult interpersonal skills", Journal of Genetic Psychology, Vol.151, n², p.211-219.

Winnicott D.W. (1989), « De la pédiatrie à la psychanalyse », Paris, Payot.

Zazzo R. (1974), « L'attachement », Paris, Delachaux et Nestlé. 